\title{
Comparison of the Cross Deleted Wigner Representation and the Matching Pursuit Distribution (Via Adaptive Signal Decomposition)
}

\author{
S. Ghofrani ${ }^{1}$ and D.C. McLernnon ${ }^{2}$ \\ ${ }^{1}$ Electrical Engineering Department, Islamic Azad University, Tehran South Branch, \\ Tehran, Iran \\ ${ }^{2}$ School of Electronic and Electrical Engineering, the University of Leeds, Leeds, UK \\ s_ghofrani@azad.ac.ir
}

\begin{abstract}
The cross-deleted Wigner representation (CDWR) suppresses the cross terms present in the Wigner distribution of a multi-component signal by decomposing the Wigner distribution (via the Gabor expansion) into terms that affect the auto- and cross-terms. The Matching Pursuit (MP) distribution is also a time-frequency representation that is devoid of cross terms. In this paper we decompose a signal by both the Gabor expansion and MP. Then the CDWR and the MP distribution are obtained. We then compare and contrast both representations (along with the original Wigner-Ville distribution (WVD)) with respect to (i) concentration/resolution, (ii) noise reduction capabilities and (iii) frequency and time resolvability.
\end{abstract}

Keywords: Wigner-Ville distribution; cross deleted Wigner representation; matching pursuit; Gabor expansion.

\section{Introduction}

Time-frequency distributions have been used extensively for non-stationary signal analysis. They describe how the frequency content of a signal changes with time. The Wigner-Ville distribution (WVD) is the best known of these [1]. For the signal $x(t)$ the WVD is:

$$
W V D_{x}(t, \omega)=\int_{-\infty}^{+\infty} x\left(t+\frac{\tau}{2}\right) x^{*}\left(t-\frac{\tau}{2}\right) e^{-j \omega \tau} d \tau
$$

where '*' represents complex conjugate and $\omega=2 \pi f$. The WVD satisfies an exceptionally large number of desirable mathematical properties, and in addition it has the best resolution among all other time-frequency distributions in the Cohen class. However, it generates cross terms when analyzing multi-component signals. These cross terms (or artefacts) mean that the distribution shows energy which does not really exist at particular time/frequency co-ordinates.

The signal decomposition is an approach to avoid the cross-terms from the timefrequency plane. In general the WVD of a decomposed signal includes two terms: the auto-Wigner and the cross-Wigner terms. The cross-deleted Wigner representation (CDWR) [2], [3] and the Matching Pursuit (MP) [4] are created by just considering 
the auto-Wigner terms. Obviously the method of signal decomposition directly affects the extracted time- frequency representation.

Dennis Gabor first suggested expanding a signal onto a set of functions that are concentrated in both the time and frequency domains [5]. Later, Mallat and Zang also suggested a similar approach [4]. But the two decomposition methods have fundamental differences. In the Gabor transform we use constant band-limited functions and project a signal onto the regular lattice network. But in MP decomposition, except for the first iteration, we find the atom most similar to the residue. Also, in MP decomposition the time and frequency bandwidth of the elementary functions can be changed in order to get the most similarity with the signal or the residue.

In this paper we decompose the signal via both the non- adaptive Gabor approach and the adaptive MP implementation. We then compute the CDWR and the MP distribution according to the extracted expansion coefficients. The CDWR is compared with the MP distribution with respect resolution, noise reduction capability, and frequency/time resolvability.

So first in section 2 the Gabor transform is explained. In section 3 MP decomposition theory is reviewed. In section 4 we analyze a non stationary synthesized signal according to the two different decomposition methods and finally in section 5 we draw some conclusions.

\section{Review of (Non-adaptive Signal Decomposition) Gabor Transform}

For signal $x(t)$ the Gabor expansion is defined as [6]:

$$
x(t)=\sum_{m=-\infty}^{+\infty} \sum_{n=-\infty}^{+\infty} c_{m, n} h_{m, n}(t)
$$

where $h_{m, n}(t)=h(t-m T) e^{j n \Omega t}, m, n \in Z$, and $T$ and $\Omega$ represent the time and frequency sampling intervals respectively. The synthesis function, $h(t)$, is subject to a unit energy constraint. The existence of (2) has been found for arbitrary $x(t)$ only for $T \Omega \leq 2 \pi$. Generally, $h_{m, n}(t)$ do not form an orthogonal basis. This means that the first problem in using the Gabor expansion is how the coefficients should be determined. The first solution is to introduce an auxiliary function. The auxiliary function, $\gamma(t)$, and the synthesis function, $h(t)$, are named biorthogonal if:

$$
<h_{m, n}, \gamma_{p, q}>=\int_{-\infty}^{+\infty} h(t-n T) \gamma^{*}(t-q T) \exp \left(j 2 \pi(m-p) \frac{t}{T}\right) d t=\delta_{n-q} \delta_{m-p} .
$$

If we can find $\gamma(t)$ then the Gabor coefficient $c_{m, n}$ can be computed as follows:

$$
c_{m, n}=<x(t), \gamma_{m, n}(t)>=\int_{-\infty}^{+\infty} x(t) \gamma_{m, n}^{*}(t) d t
$$

where $\gamma(t)$ is also called the analysis function. The second problem in using the Gabor expansion is whether the coefficients reflect the signal behavior or not. For this 
purpose the synthesis function and the analysis function must to be localized in both the time and frequency domains and have the most possible similarity to each other. Gabor chose the Gaussian pulse because it is a unique function which exhibits maximum concentration in both time and frequency domains.

Now the WVD of the decomposed signal in (2) is written as:

$$
W V D_{x}(t, \omega)=\sum_{m, n=-\infty}^{+\infty}\left|c_{m, n}\right|^{2} W_{h_{m, n}}(t, \omega)+\sum_{m, n=-\infty}^{+\infty} \sum_{m^{\prime}, n^{\prime}=-\infty}^{+\infty} c_{m, n} c_{m^{\prime}, n^{\prime}}^{*} W_{h_{m, n} h_{m^{\prime}, n^{\prime}}}(t, \omega)
$$

where $W_{h_{m, n}}(t, \omega)$ and $W_{h_{m, n} h_{m^{\prime}, n^{\prime}}}(t, \omega)$ are the auto- and cross-WVD of the appropriate synthesis functions.

In this way, Qian, and Morris [2] introduced the time- frequency distribution series while Kadambe and Orr suggested [3] that the double sum in (5) corresponds to the cross-terms of the WVD. So keeping only the first term in (5), they defined the CDWR as follows:

$$
C D W R_{x}(t, \omega)=\sum_{m, n=-\infty}^{+\infty}\left|c_{m, n}\right|^{2} W_{h_{m, n}}(t, \omega)
$$

\section{Review of (Adaptive Signal Decomposition) Matching Pursuit}

Mallat and Zhang [4] proposed an adaptive signal decomposition method. This method is based on a dictionary that contains a family of elementary functions or time-frequency atoms. The decomposition of a signal is performed by projecting the signal over the function dictionary and then selecting the atoms which can best match the local structure of the signal. So we compute a linear expansion of $x(t)$ over a set of elementary functions selected from the dictionary in order to best match its inner structures. The MP decomposition after $M$ iterations can be written as follows:

$$
x(t)=\sum_{n=0}^{M-1} c_{n} g_{\gamma_{n}}(t)+R^{M} x(t)
$$

where $x(t)$ is the decomposed signal and $R^{M} x(t)$ is the residue after $M$ times signal decomposition. By letting $R^{0} x(t)=x(t)$, then the MP algorithm decomposes the residue at each stage. Thus the original signal is projected onto a sum of elementary functions which are chosen to best match its residues. Now $g_{\gamma_{n}}(t)$ is the time-frequency atom that belongs to the dictionary and which satisfies the unit-norm requirement - i.e. $\int_{-\infty}^{+\infty}\left|g_{\gamma_{n}}(t)\right|^{2} d t=1$; the coefficient $c_{n}=<R^{n} x(t), g_{\gamma_{n}}(t)>=\int_{-\infty}^{+\infty} R^{n} x(t) \cdot g_{\gamma_{n}}^{*}(t) d t$ is the inner product of the functions $R^{n} x(t)$ and $g_{\gamma_{n}}(t)$; and $\gamma_{n}$ refers to the atom's parameter set. So when the number of iterations is infinitive, then the residue will be zero, and we can say 


$$
\operatorname{Lim}_{M \rightarrow \infty} R^{M} x(t)=0 \Rightarrow x(t)=\sum_{n=0}^{+\infty} c_{n} g_{\gamma_{n}}(t)
$$

where $c_{n}$ is complex. The WVD of the decomposed signal is thus written as:

$$
W V D_{x}(t, \omega)=\sum_{n=0}^{+\infty}\left|c_{n}\right|^{2} W_{g_{\gamma_{n}}}(t, \omega)+\sum_{n=0}^{+\infty} \sum_{\substack{m=0 \\ m \neq n}}^{+\infty} c_{n} c_{m}^{*} W_{g_{\gamma_{n}} g_{\gamma_{m}}}(t, \omega)
$$

where $W_{g_{\gamma_{n}}}(t, \omega)$ and $W_{g_{\gamma_{n}} g_{\gamma_{m}}}(t, \omega)$ are the auto- and cross-WVD of the appropriate atoms. Mallat and Zhang [4] suggested that the double sum in (9) corresponds to the cross terms of the WVD. So keeping only the first term in (9), they defined the MP distribution as follows:

$$
E_{x}(t, \omega)=\sum_{n=0}^{+\infty}\left|c_{n}\right|^{2} W_{g_{\gamma_{n}}}(t, \omega)
$$

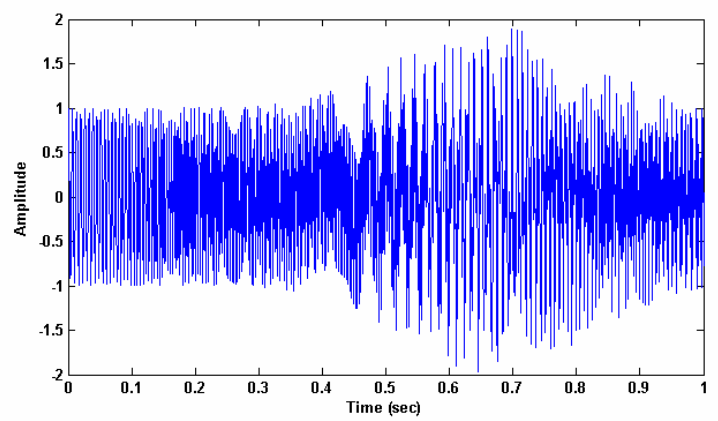

Fig. 1. The real part of the non-stationary signal in (11)

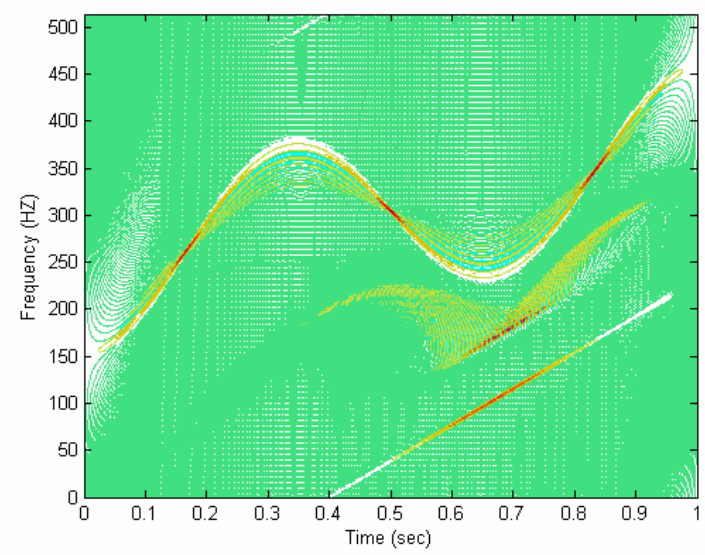

Fig. 2. The Discrete WVD of (11), with excellent resolution and negligible cross terms 
This new distribution, $E_{x}(t, \omega)$, can now be interpreted as an energy density function of $x(t)$ in the time-frequency plane. Mallat and Zhang claimed that this new distribution does not include any cross terms.

\section{Analyzing Synthetic Test Signals}

Now we consider the following signal:

$$
x(t)=e^{j\left(-60 \sin (3 \pi t)+400 t^{2}+1500 t\right)}+e^{-25(t-0.67)^{2}} e^{j 1200(t-0.4)^{2}}
$$

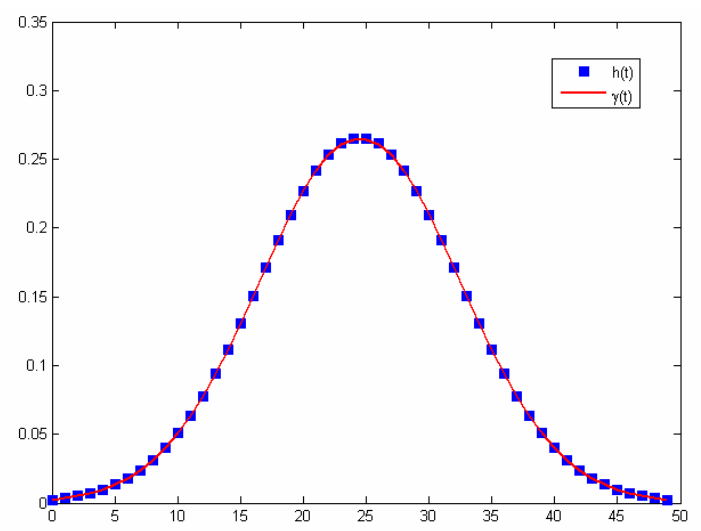

Fig. 3. The biorthogonal analysis and synthesis functions, both with same length $L=50$

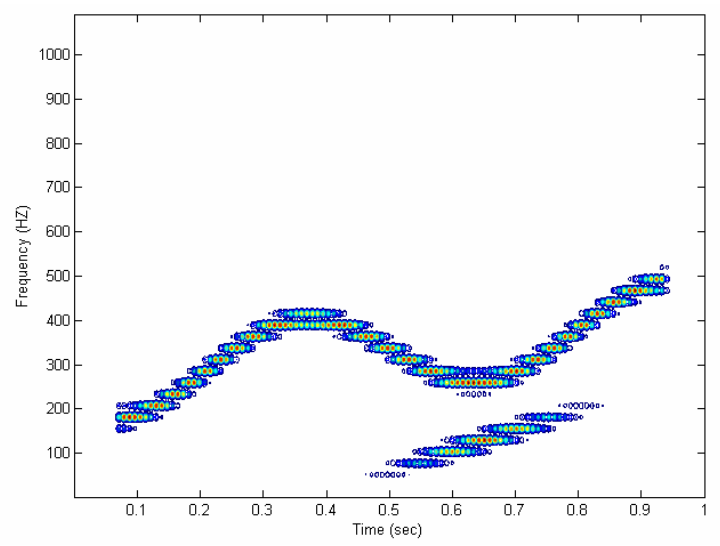

Fig. 4. The CDWR of $x(t)$ based on the Gabor expansion. The number of computed Gabor coefficients is 4210 . 


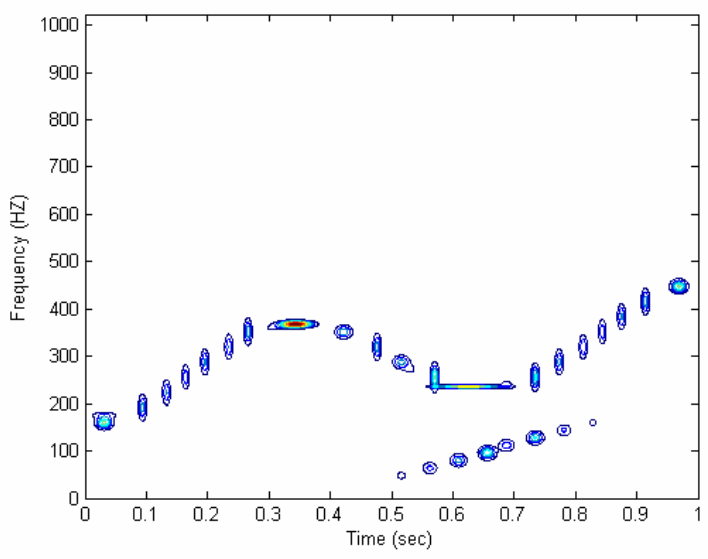

Fig. 5. The MP distribution of $x(t)$ based on Gaussian atoms. The number of iterations is 32 .

This test signal (also previously used in [7]) is sampled at 1024 points over the time interval [ $\left[\begin{array}{ll}0 & 1\end{array}\right]$ and is plotted in Fig. 1. Its discrete WVD is then displayed in Fig. 2. We use an efficient algorithm [8] to obtain the discrete WVD where the window length has been chosen to give the best visual interpretation.

Now we decompose the signal via Gabor expansion theory and then construct the CDWR (see (6)). The major problem with the Gabor expansion is how to find the biorthogonal analysis function. In this paper we consider the orthogonal-like Discrete Gabor Transform (DGT) for an infinite signal [9] - as the real signal is usually very long sequence. In the orthogonal-like DGT, the Gabor coefficients can be thought of as the measure of similarity between the underlying signal, $x(i)$, and the individual basis functions, $\left\{h_{m, n}\right\}$. Thus it will reflect the signal's local behavior as long as the given synthesis window, $h(i)$, is indeed localized. So we try to obtain the biorthogonal function, $\gamma(i)$, that has the most similarity with $h(i)$. We restricted the length of $h(i)$ and $\gamma(i)$ to be the same. As long as the synthesis window function, $h(i)$, is localized, the orthogonal-like DGT will well reflect signal local behaviors because $c_{m, n}$ is very close to the inner product of $x(i)$ and $h_{m, n}$. Because the Gaussian window has the property of optimal concentration it is therefore very useful for time frequency analysis. We have chosen the synthesis window, $h(i)$, to be Gaussian with length $L=50$ and variance $\sigma^{2}=\frac{N \Delta M}{2 \pi}$, where $\Delta M$ denotes the discrete time sampling step and $N$ is equal to the number of sampling points in frequency. The amplitude of the synthesis function is chosen as $A=\frac{1}{\sqrt[4]{\pi \sigma^{2}}}$ to satisfy the unit norm requirement. The optimum parameters for DGT have been chosen as: 


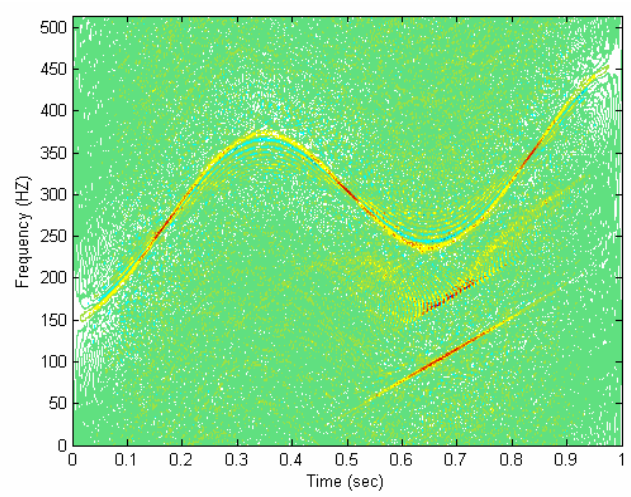

(a)

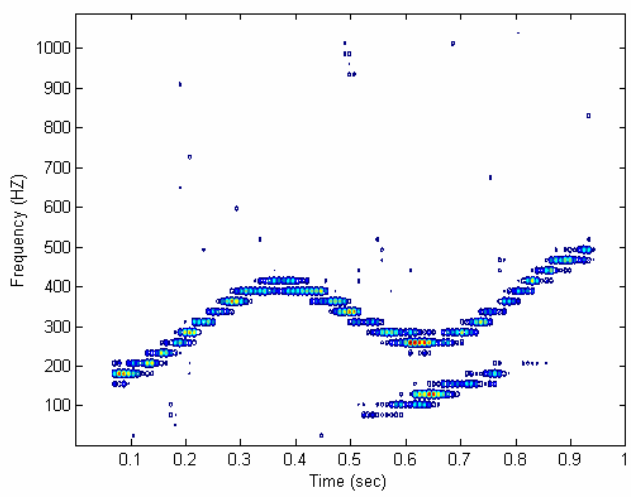

(b)

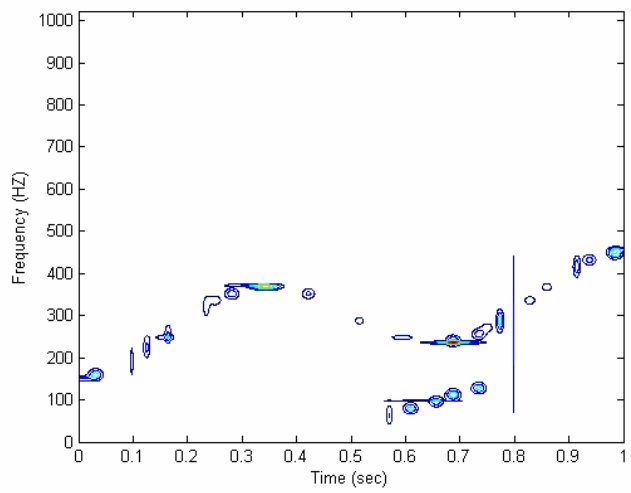

(c)

Fig. 6. (a) The Discrete WVD of the signal in (11) to which white Gaussian noise has been added ( $\mathrm{SNR}=0 \mathrm{~dB}$ ); (b) the CDWR - number of Gabor coefficients is 4210; (c) the MP distribution - number of algorithm iterations is 32 


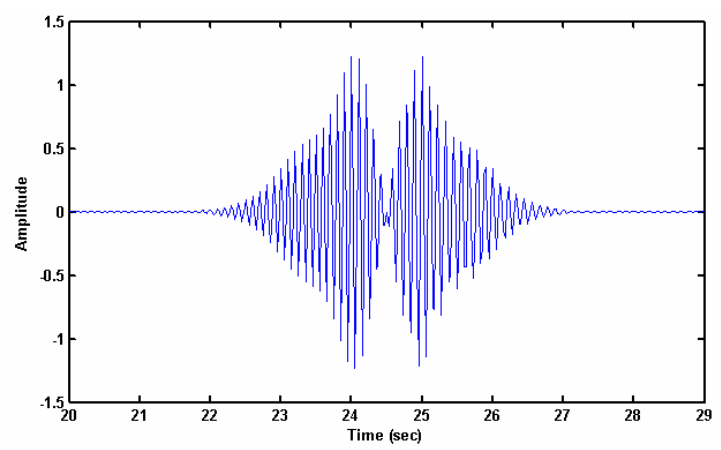

Fig. 7. The real part of the two component signal, in (14)

$\Delta M=10, N=40, \Delta N=\frac{L+L_{x}}{N}$. Note that $L_{x}=1024$ refers to the signal length, and $\Delta N$ denotes the discrete frequency sampling steps. Therefore in order to implement the DGT we require $M \times N=107 \times 40$ coefficients, where $M$ is equal to the number of sampling points in time and determined according to the signal lengths and $\Delta M$. So the synthesis window is selected to be Gaussian and the biorthogonal analysis function that has the most similarity to the synthesis function is determined according to the algorithm described in [9]. Fig.3 shows the calculated analysis and synthesis functions. Now we try to decompose the signal and obtain the CDWR. According to the $\mathrm{M}$ and $\mathrm{N}$ values, we have to project the signal on the $M \times N=4210$ Gaussian functions. The whole Gaussian functions have the same time and frequency bandwidth and just have been shifted in time and or in frequency. The CDWR is shown in Fig. 4. No cross terms are there while decreasing the resolution in comparison with the discrete WVD, Fig. 2, is obviously. So we have also decomposed the signal according MP by using the Gaussian atoms. It is well known that the Gaussian atom is unique in the sense that it has the greatest "concentration" in both the time and frequency domains. Such an atom is:

$$
g_{\gamma_{n}}(t)=\frac{1}{\sqrt{s_{n}}} g\left(\frac{t-u_{n}}{s_{n}}\right) e^{j \zeta_{n} t}
$$

where $g(t)=2^{1 / 4} e^{-\pi t^{2}}$ and $\gamma_{n}=\left(s_{n}, u_{n}, \zeta_{n}\right)$ represents the atom's parameters set. The parameter $s_{n}$ controls the envelope width of $g_{\gamma_{n}}$. The parameters $u_{n}$ and $\zeta_{n}$ are respectively the temporal placement and the frequency variable. The parameter set are all real. In addition, $s_{n}$ is also positive. As we use the Gaussian dictionary for the MP signal decomposition, the index $n$ refers to the different atoms that exist in the dictionary. Now, suppose that a signal $x(t)$ is decomposed adaptively by employing the Gaussian elementary functions as in (8). It can be demonstrated that the MP distribution with the Gaussian atom is:

$$
E_{x}(t, \omega)=2 \sum_{n=0}^{+\infty}\left|c_{n}\right|^{2} \tilde{H}\left(\frac{t-u_{n}}{s_{n}}\right) \tilde{F}\left(s_{n}\left(\omega-\zeta_{n}\right)\right)
$$




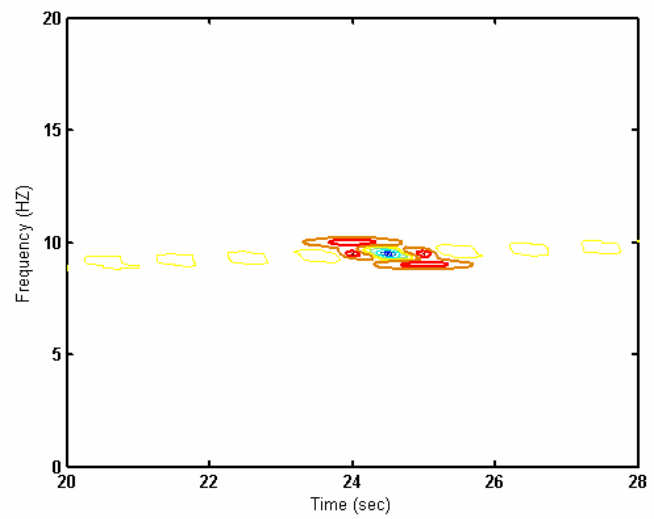

(a)

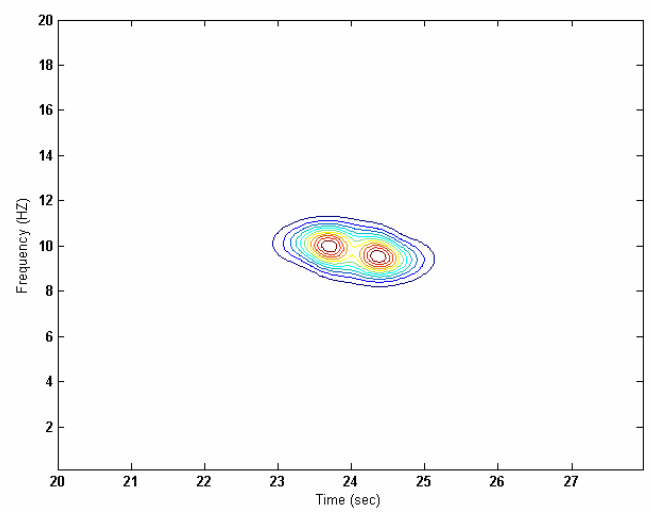

(b)

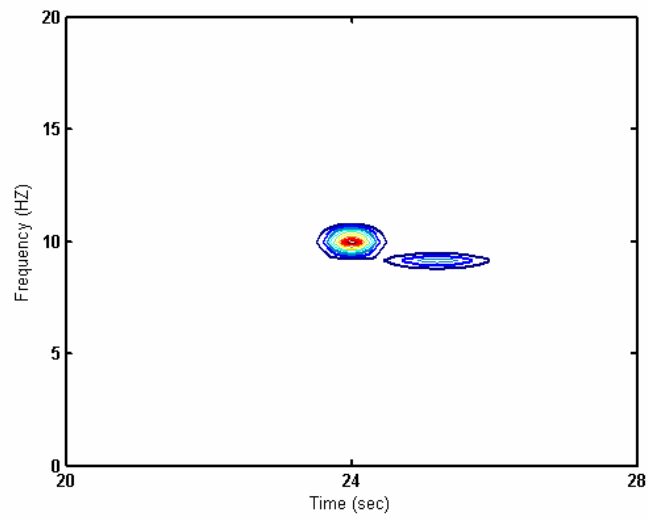

(c)

Fig. 8. (a) The Discrete WVD of the two-component signal in (14); (b) the CDWR; and (c) the MP distribution. It can be seen that the time and frequency resolvability of the MP is better than both the discrete WVD and the CDWR. 
with $\tilde{H}(t)=e^{-2 \pi t^{2}} \tilde{F}(\omega)=e^{-\frac{1}{2 \pi} \omega^{2}}$. Although we have explained the MP decomposition theory for Gaussian elementary functions for the continuous time signals, it should be implemented for processing discrete time signals and so we use the algorithm in [10]. Thus we have decomposed the signal $x(i)$ and obtained the best 32 Gaussian atoms. The MP distribution has been evaluated and is illustrated in Fig. 5. As we can see, there are no significant cross terms. The MP distribution has also better resolution compared to the CDWR. In addition the 4210 coefficients must be computed via Gabor decomposition while the number of MP algorithm iterations is set to be just 32 . We also added white Gaussian noise $(\mathrm{SNR}=0 \mathrm{~dB})$ to the signal in (11) and determined the CDWR according to the Gabor coefficients and the MP distribution via using the Gaussian elementary function, as well as the discrete WVD. These results are shown in Fig. 6. Note that the noise is spread in time-frequency plane for both the discrete WVD and the CDWR. Note that the noise affects the choice of the optimum atoms for the MP distribution. This feature may be interpreted as the MP noise reduction capability.

For testing resolvability, we consider the following $x(t)$ :

$$
x(t)=\sqrt[4]{\frac{2}{\pi}} \cdot e^{-(t-24)^{2}} e^{j 20 \pi(t-24)}+\sqrt[4]{\frac{2}{\pi}} \cdot e^{-(t-25)^{2}} e^{j 18 \pi(t-25)} .
$$

The signal is sketched in Fig. 7. It includes of two Gaussian components. The first term is concentrated at $(24,10)$ in the time-frequency space and the second term is concentrated at $(25,9)$. The results are shown in Fig. 8. While the discrete WVD cannot distinguish the two terms at all, the CDWR shows one complex term with the two seeds. On the other hand the MP distribution detects and shows the two Gaussian components clearly. This feature is because of its capability to adapt both the atom's time and the frequency bandwidth in MP. So the adaptive decomposition shows a better performance with respect to the resolvability.

\section{Conclusion}

Although the potential of the WVD has long been recognized in signal processing, its applications are limited mainly due to the cross-terms interference problem. An alternative to the WVD is other time-frequency signal decompositions. One method is the constant band-width analysis achieved by the Gabor transform and the adaptive decomposition, MP. In this paper we have used the discrete WVD, CDWR, and the MP distribution for analyzing the non-stationary signals. The three time frequency representations are compared as regards resolution, noise reduction capability, and resolvability. Future work will concentrate on comparing the three time frequency representations according to both instantaneous frequency estimation and instantaneous bandwidth estimation.

\section{References}

1. Cohen, L.: Time-Frequency Distribution- A Review. Proceedings of the IEEE 77(7), 941980 (1989)

2. Qian, S., Morris, J.: Wigner distribution decomposition and cross terms deleted representation. IEEE Trans. Sig. Proc. 27(2), 125-144 (1992) 
3. Kadambe, S., Orr, R.: Instantaneous frequency estimation using the cross- term deleted Wigner representation (CDWR). In: TFTS 1996, pp. 289-292 (1996)

4. Mallat, S.G., Zhang, Z.: Matching pursuit with time-frequency dictionaries. IEEE Trans. Signal Processing 41(12), 3397-3415 (1993)

5. Gabor, D.: Theory of communication. J. Inst. Electr. Eng. 93(3), 429-457 (1946)

6. Qian, S.: Introduction to time- frequency and wavelet transforms. Prentice-Hall, Englewood Cliffs (2002)

7. Qian, S., Stankovic, L.: Analysis of noise in time- frequency distributions. IEEE Signal Processing Letters 9(9) (2002)

8. Boashash, B., Black, P.J.: An efficient real-time implementation of the Wigner-Ville distribution. IEEE Trans. ASSP 35(11), 1611-1616 (1987)

9. Qian, S., Chen, D.: Discrete Gabor Transform. IEEE Trans. Sig. Proc. 41(7), 2429-2438 (1993)

10. Ferrando, S.E., Kolasa, L.A.: Algorithm 820: a flexible implementation of matching pursuit for Gabor functions on the interval. ACM Trans. Mathematics Software 28(3), 337 $353(2002)$ 